\title{
CONSTRUCTION OF A GROUPLESS AND HEADLESS TRIAD SYSTEM ON 31 ELEMENTS*
}

\author{
BY H. S. WHITE
}

No doubt the most interesting triad systems are those which admit a large group of substitutions, for example, those of the Heffter headless type, $\dagger$ admitting a cyclic substitution on all the elements. But on a sufficiently large set of elements systems can be constructed which have smaller groups or even no group. $\ddagger$ The structure of such systems is worth recording for the same reason as, in the theory of algebraic plane curves, not only those with no singular points, but also those with all admissible singularities and actual aggregates of singularities demand enumeration.

The least number of elements in a groupless system is fifteen. $\S$ For thirty-one elements, Reiss's method can be adapted to yield a triad system with no group, $\|$ but a system built by that method has necessarily a "head", namely a triad (sub-)system on fifteen, a $\Delta_{15}$, which itself has no group. This class of $\Delta_{31}$ 's, millions in number, were at their first appearance a surprise, since groupless systems previously known were also headless. But the obvious question was still unanswered: is there any $\Delta_{31}$ which is both groupless and headless? I shall here exhibit one such by actual construction, and describe the application of the method of sequences and indices by which it is proved to be both groupless and headless. This method is of course tedious,arousing the wish that some clearer insight and more sweeping form of argument might be attained.

* Presented to the Society, September 11, 1930.

$\dagger$ L. Heffter, Ueber Tripelsysteme, Mathematische Annalen, vol. 49 (1897), p. 101.

$\ddagger \mathrm{H}$. S. White, The multitude of triad systems on thirty-one elements, Transactions of this Society, vol. 16 (1915), pp. 13-19.

$\S$ See reference to DePasquale and Brunel in article by F. N. Cole, The triad systems of thirteen elemen's Transactions of this Society, vol. 16 (1913), p. 1.

\| H.S. White, loc cit 
We will take as a starting point a certain $\Delta_{15}$ classified by its discoverer, the late Professor F. N. Cole, ${ }^{*}$ as VI, 2, and by Miss L. D. Cummings $\dagger$ as Groupless No. 36. It will be used once with numbers as elements, the integers from 1 to 15 ; and a second time, according to the Reiss method, with letters from $a$ to $r$, omitting $i$, and Reiss's splicing element shall be denoted by $s$. To forestall a group of order 2, I shall derange arbitrarily the columns of the second set, reversing by threes, that is, exchanging, before splicing, columns headed by $s a$ and $s c$, also $s d$ and $s f$, etc. There results a groupless $\Delta_{31}$ having two heads (the two $\Delta_{15}$ 's). Arranged under the elements of the first of these $\Delta_{15}$ 's, in 15 columns, it is the following array. One misprint has been corrected, Cole's triad 81115 being changed to 91115 as of course it must have been in his MS.

\begin{tabular}{|c|c|c|c|c|c|c|c|c|c|c|c|c|c|c|c|}
\hline 1 & & 2 & & 3 & & 4 & & 5 & & 6 & & 7 & & 8 & \\
\hline 2 & 3 & 1 & 3 & 1 & 2 & 1 & 5 & 1 & 4 & 1 & 7 & 1 & 6 & & 9 \\
\hline 4 & 5 & 4 & 6 & 4 & 8 & 2 & 6 & 2 & 7 & 2 & 4 & 2 & 5 & & 10 \\
\hline 6 & 7 & 5 & 7 & & 12 & 3 & 8 & 3 & 12 & & 14 & & 11 & & 4 \\
\hline 8 & 9 & 8 & 10 & 6 & 14 & 7 & 15 & 6 & 9 & 5 & 9 & 4 & 15 & & 15 \\
\hline 10 & 11 & 9 & 12 & 7 & 11 & 9 & 10 & 8 & 15 & 8 & 11 & 8 & 13 & & 11 \\
\hline 12 & 13 & 11 & 14 & 9 & 13 & 11 & 12 & 10 & 14 & 10 & 13 & 9 & 14 & & 13 \\
\hline 14 & 15 & 13 & 15 & 10 & 15 & 13 & 14 & 11 & 13 & 12 & 15 & 10 & 12 & 12 & 14 \\
\hline$s$ & $c$ & $s$ & $b$ & $s$ & $a$ & $s$ & $f$ & $s$ & $e$ & $s$ & $d$ & $s$. & $j$ & $s$ & $h$ \\
\hline$a$ & $b$ & $a$ & $c$ & $b$ & $c$ & $a$ & $g$ & $a$ & $d$ & $a$ & $e$ & $a$ & $h$ & $a$ & $j$ \\
\hline$d$ & $h$ & $d$ & $f$ & $d$ & $e$ & $b$ & $d$ & $b$ & $g$ & $b$ & $f$ & $b$ & $m$ & $b$ & $k$ \\
\hline$e$ & $m$ & $e$ & $g$ & $f$ & $g$ & $c$ & $p$ & $c$ & $m$ & $c$ & $h$ & $c$ & $n$ & $c$ & $d$ \\
\hline$f$ & $p$ & $h$ & $k$ & $h$ & $j$ & $e$ & $j$ & $f$ & $j$ & $g$ & $r$ & $d$ & $k$ & $e$ & $r$ \\
\hline$g$ & $l$ & $j$ & $m$ & $k$ & $l$ & $h$ & $l$ & $h$ & $r$ & $j$ & $k$ & $e$ & $f$ & $f$ & $l$ \\
\hline j & $n$ & $l$ & $p$ & $m$ & $n$ & $k$ & $n$ & $k$ & $p$ & $l$ & $m$ & $g$ & $p$ & $g$ & $n$ \\
\hline$k$ & $r$ & $n$ & $r$ & $p$ & $r$ & $m$ & $r$ & $l$ & $n$ & $n$ & $p$ & $l$ & $r$ & & $p$ \\
\hline 9 & & \multicolumn{2}{|c|}{10} & \multicolumn{2}{|c|}{11} & \multicolumn{2}{|c|}{12} & \multicolumn{2}{|c|}{13} & \multicolumn{2}{|c|}{14} & \multicolumn{2}{|c|}{15} & \multicolumn{2}{|c|}{$r$} \\
\hline & 8 & \multicolumn{2}{|c|}{111} & \multicolumn{2}{|c|}{$\begin{array}{ll}1 & 10\end{array}$} & \multicolumn{2}{|c|}{113} & & 12 & \multirow{2}{*}{\multicolumn{2}{|c|}{$\begin{array}{ll}1 & 15 \\
2 & 11\end{array}$}} & & 14 & \multicolumn{2}{|c|}{$1 k$} \\
\hline 2 & 12 & & & 14 & 2 & 9 & & 15 & & & & 13 & & $n$ \\
\hline 3 & & & & \multicolumn{2}{|c|}{37} & 3 & 5 & 3 & 9 & \multicolumn{2}{|c|}{36} & & 10 & \multicolumn{2}{|c|}{$3 p$} \\
\hline
\end{tabular}

* F. N. Cole, Complete classification of the triad systems on fifteen elements, Memoirs of the National Academy of Sciences, vol. 14 (1919), Second Memoir, Part 4.

$\dagger$ Louise D. Cummings, in the Memoir just cited, Part 5. 


$\begin{array}{rrrrrrrrrrrrrrrr}4 & 10 & 4 & 9 & 4 & 12 & 4 & 11 & 4 & 14 & 4 & 13 & 4 & 7 & 4 & m \\ 5 & 6 & 5 & 14 & 5 & 13 & 5 & 15 & 5 & 11 & 5 & 10 & 5 & 8 & 5 & h \\ 7 & 14 & 6 & 13 & 6 & 8 & 7 & 10 & 6 & 10 & 7 & 9 & 6 & 12 & 6 & g \\ 11 & 13 & 7 & 12 & 9 & 15 & 3 & 14 & 7 & 8 & 8 & 12 & 9 & 11 & 7 & l \\ s & g & s & m & s & l & s & k & s & r & s & p & s & n & 8 & e \\ a & f & a & n & a & k & a & l & a & p & a & r & a & m & 9 & d \\ b & e & b & j & b & p & b & h & b & n & b & l & b & r & 10 & f \\ c & l & c & e & c & g & c & r & c & k & c & f & c & j & 11 & j \\ d & r & d & l & d & m & d & j & d & g & d & n & d & p & 12 & c \\ h & n & f & r & e & n & e & p & e & h & e & k & e & l & 13 & s \\ j & p & g & k & f & h & f & n & f & m & g & j & f & k & 14 & a \\ k & m & h & p & j & r & g & m & j & l & h & m & g & h & 15 & b\end{array}$

To eradicate the two heads, I choose the column of triads containing 1 , and that containing $r$, which latter has been collected from the first fifteen and placed after them in the above table. Between these columns the tetrad relation* occurs three times. I select the triads $167,1 \mathrm{gl}$, and $r 6 g, r 7 l$, and replace them by $16 g, 17 l$, and $r 67, r g l$. After this alteration (which requires corresponding alterations in columns under 6 and 7 ), the upper half and lower half of that $15 \times 15$ array no longer form mutually exclusive closed sets, letters in one and integers in the other, for there are now three mixed triads containing one letter each; that is, six mixed pairs in the columns 1, 6, 7. This fact enables me to cut corners in the argument. All triads of the $\Delta_{31}$ are listed already in these 15 columns, but for detailed study the 16 additional columns are written out, but not in full. This part need not be given in all detail, since a bare outline will enable anyone to repeat it. I have formed the sequences and listed the indices, complete for columns 1 to 9 inclusive, and enough others so that at least nine indices are listed for triads which contain any particular element. This minimum proves to be sufficiently large for the present purpose.

Verification. (1) There is no $\Delta_{15}$ contained in this $\Delta_{31}$. If there were, its 15 elements would occur each in 7 triads, and the sequences derived from those triads would constitute a closed system, every pair of elements present occurring twice, in opposite orders, with indices totaling 14 in each column concerned. For

* Memoir cited, p. 73 and p. 70. "Quadrangular" = "Tetrad". 
the complete $\Delta_{31}$ the indices total 28 for each column. I examine therefore the sequences belonging to each triad separately, noting how many are related by the appearing of one pair in two sequences. Where the index total of related sequences forming a closed set under a triad is 16 or more, neither that set nor the remainder under that triad can belong to a $\Delta_{15}$. Such is the case with at least 9 of the triads in every column; and we need examine no further, since $15-9=6<7$, and it is therefore certain that no element can be found in 7 triads, to form a closed system with the 14 associated elements.

(2) There is no substitution, aside from the identity, which can transform this $\Delta_{31}$ into itself. Any such substitution would replace at least one element by a different one; and the set of indices associated with the former would necessarily be identical with the set belonging to the latter. Now having the complete table of indices for nine columns, I compare them among themselves and find no two identical. Then I compare the partial set that has been formed for each of the remaining 22 columns with each of the first 9, and find in every partial column at least one index that does not occur in any complete column. Lastly, I examine, for every two of the 22 partial columns, the twice nine indices which they exhibit. If, when completed, the two sets of columns would become identical, the partial columns ought to show at least 4 indices repeated $(18-14=4)$, or the equivalent. But this does not happen in any two partial columns of the 22 , and we may omit the labor of carrying these columns to completion. The quality of grouplessness is fully certified.

Let me repeat for the benefit of anyone who may wish to check this proof : in verifying (1) it was frequently necessary to cite the fact that, after the tetrad alteration, neither integers alone nor letters alone are, in this array, a closed set.

It remains an interesting question whether 31 is the least number of elements above 15 for which a groupless and headless triad system can exist.

Vassar College 